ULB-TH-01/33

KUL-TF-2001/23

\title{
Comments on duality-symmetric theories
}

\author{
Xavier Bekaert ${ }^{1}$ and Sorin $\mathrm{Cucu}^{2}$ \\ ${ }^{1}$ Physique Théorique et Mathématique, Université Libre de Bruxelles, \\ Campus Plaine C.P. 231, B-1050 Bruxelles, Belgium \\ Fax : +32-2-650.58.24 \\ xbekaert@ulb.ac.be \\ ${ }^{2}$ Instituut voor Theoretische Fysica, Katholieke Universiteit Leuven, \\ Celestijnenlaan 200D, B-3001 Leuven, Belgium \\ Fax. +32-16-32.79.86 \\ sorin.cucu@fys.kuleuven.ac. be
}

\begin{abstract}
We briefly review the basics of electric-magnetic duality symmetry and their geometric interpretation in the M-theory context. Then we recall some no-go theorems that prevent a simple extension of the duality symmetry to non-Abelian gauge theories.
\end{abstract}

\section{Introduction}

Duality symmetry has a rather old history, going back to the birth of Maxwell equations. It seems likely that the symmetric role played by the electric and the magnetic fields in the laws of electromagnetism, has been one of Maxwell's motivations to modify Ampère's law by adding the "displacement current" term $\frac{\partial \mathbf{E}}{\partial t}$, which was not sanctioned by experiment at that time. Maxwell was known to have an aesthetic appreciation for mathematical structures. In this light, it is reasonable to endorse the conclusion of Roger Penrose: "It would seem that the symmetry of these equations and the aesthetic appeal that this symmetry generated must have played an important role for Maxwell in his completion of these equations" [1].

As a whole, sourceless Maxwell equations are invariant under the discrete transformation

$$
\mathrm{E} \rightarrow \mathrm{B}, \quad \mathrm{B} \rightarrow-\mathrm{E} .
$$

This is called the electric-magnetic (EM) duality symmetry. The energy-momentum tensor is also left invariant under this transformation. This $\mathbb{Z}_{2}$-duality symmetry (11) of sourceless Maxwell theory has been generalized in various ways. First of all, the discrete duality symmetry extends to a continuous $S O(2)$ symmetry. Secondly, if we introduce a $\theta$-term, the EM-duality symmetry group is enhanced from $S O(2)$ to $S L(2, \mathbb{R})$ group.

\footnotetext{
${ }^{1}$ Talk given by X.B. at the RTN workshop "The Quantum Structure of Spacetime and the Geometric Nature of Fundamental Interactions", Corfu, 13-20 Sept 2001.
} 
Thirdly, we can promote it from an invariance of linear electrodynamics (i.e. Maxwell theory) to non-linear electrodynamics (e.g. Born-Infeld theory) [2, 3]. An other possibility is to introduce both electric and magnetic sources, in which case the EM-duality symmetry is preserved if we also "rotate" the sources in an appropriate way. Let us mention the important fact that, at the quantum level, electric and magnetic charges have to satisfy quantization conditions $[4,5]$. In the quantum theory, it follows that the $S L(2, \mathbb{R})$ duality symmetry group is broken to $S L(2, \mathbb{Z})$ (if $\theta$ is constrained to vanish, the $S O(2)$ symmetry is reduced to the previous discrete $\mathbb{Z}_{2}$ transformation). Four-dimensional Maxwell theory is an Abelian gauge field theory with one vector field. The EM-duality symmetry holds in general for Abelian $p$-form theories in dimension 2( $p+1)$ [5]. In addition, people have considered the case of several Abelian gauge fields, in which case the duality symmetry is further enlarged [3]. Recently, the Hodge duality symmetry has been applied to linearized gravity and higher spin gauge field theories [6]. To conclude this (non-exhaustive) list, let us add that type IIB superstring theory is expected to have an $S L(2, \mathbb{Z})$ symmetry, the so-called $S$-duality [7].

The next sections will briefly review some basics of EM-duality symmetry. Making manifest any symmetry of a theory is always highly valuable for technical and conceptual issues. In fact, it is possible to raise EM-duality symmetry to a manifest symmetry of the action. In section 3 we deal with this question. Glancing at the huge list of possible extensions of EM-duality symmetry, it is tempting try to generalize EM-duality to nonAbelian gauge theories. M-theory even brings some argument to believe that such a generalization should exist in a way or another (section 1 ). Unfortunately, some no-go theorems prevent such an extension if some (not so restrictive) hypotheses are satisfied. We recall them in the 5th section together with their hypotheses.

\section{Some basics of EM-duality symmetry}

In order to make higher dimensional generalizations more transparent, we will reformulate Maxwell equations in terms of differential forms. To proceed, we define the electric and magnetic fields as components] of the field strength two-form $F$. The sourceless Maxwell equations can be rewritten as

$$
d\left(\begin{array}{c}
F \\
* F
\end{array}\right)=0
$$

where $*$ is the Hodge dual. In other words, the field strength $F$ is a harmonic two-form.

Usually, the Poincaré lemma is used to derive from the Bianchi identity the existence of a potential vector $A$ such that $F=d A$. Then it is possible to deduce the field equation $d * F=0$ from an action principle (in all what follows we will omit sources). Alternatively, the Poincaré lemma can be applied to the field equation to get $* F=d \tilde{A}$. Then the Bianchi identity will be obtained as the equation of motion of an action depending on $\tilde{A}$, dual to the Maxwell action.

The sourceless Maxwell equations are manifestly invariant under a duality rotation:

$$
\left(\begin{array}{c}
F \\
* F
\end{array}\right) \rightarrow\left(\begin{array}{cc}
\cos \alpha & \sin \alpha \\
-\sin \alpha & \cos \alpha
\end{array}\right)\left(\begin{array}{c}
F \\
* F
\end{array}\right)
$$

Let us make here the important remark that this duality transformation is a non-local map in terms of the gauge field $A$. For an infinitesimal transformation, we have $\delta A=$

\footnotetext{
${ }^{2}$ The electric and magnetic field are the time component of, respectively, the field strength and its Hodge dual: $E^{i}=F^{0 i}$, $B^{i}=\frac{1}{2} \epsilon^{i j k} F_{j k}$.
} 
$d^{-1}(* F) \delta \alpha$, where $d^{-1}$ stands for the non-local operator which is the inverse of the differential $d$.

The first obvious generalization is to consider the field strength $F$ to be a $(p+1)$-form in $D$ spacetime dimensions. Then if we want $F$ and its dual $* F$ to have the same rank, the dimension is restricted to $D=2(p+1)$. Naively, the sourceless Maxwell equations (2) are left invariant by any transformation (3) where $R \in G L(2, \mathbb{R})$. But we have to take into account the relation $*^{2} F=(-)^{p} F$ (Minkowskian spacetime signature). Furthermore, if we ask for the invariance of the energy-momentum tensor, we get that the global symmetry group is $S O(2)$ for $D=0 \bmod 4$, and $\mathbb{Z}_{2}$ for $D=2 \bmod 4$ [5]. In the following, we will label as duality-symmetric theories only the first case.

\section{Duality-symmetric theories}

Physicists aimed to make EM-duality symmetry manifest in the action itself. Efforts in this direction have been undertaken also before (see for instance [8, 9]), but more substantial results have been achieved during the last decade after the connections with supergravity and string theory have been pointed out. As a result one counts different formulations from quadratic but non-covariant versions [9, 10], to quadratic and covariant actions but with an infinite number of auxiliary fields [11], or to non-polynomial Lagrangians with manifest space-time symmetry [12], the so-called PST model[]. Obviously, it appears that (in any of the formulations) a high price has to be paid in order to implement the duality symmetry at the level of the action.

One of the basic ingredients of the duality-symmetric formulation resides in increasing the number of gauge fields (doubling in that case) to make global symmetries manifest. At the same time, the number of gauges symmetries increases in such a way that the theory possesses the same number of physical degrees of freedom.

Formally, we can solve sourceless Maxwell equations using naively the Poincaré lemma and obtain

$$
\left(\begin{array}{c}
F \\
* F
\end{array}\right)=d\left(\begin{array}{c}
A^{1} \\
A^{2}
\end{array}\right) \equiv\left(\begin{array}{c}
F^{1} \\
F^{2}
\end{array}\right) .
$$

The electric and magnetic variables are now on the same footing. In dimensions $D=$ $0 \bmod 4$, the Hodge square relation imposes for consistency

$$
\epsilon^{a b} F^{b}=* F^{a}, \quad(a=1,2) .
$$

As we can see, the field equation now follow from the Bianchi identity. Therefore, the idea is simply to obtain this self-duality equation as e.o.m. derived from an action principle (this gives some hint that a relation should exist between EM-duality symmetry and chiral forms, as will be explained in the next section). This has been achieved in [9]-[12]. The EM-duality rotation (3) written in terms of the new variables is

$$
\left(\begin{array}{c}
A^{1} \\
A^{2}
\end{array}\right) \rightarrow R\left(\begin{array}{c}
A^{1} \\
A^{2}
\end{array}\right), \quad R \in S O(2) .
$$

The e.o.m. (5) is manifestly invariant under this transformation. Furthermore, a nice feature of duality-symmetric formulation is that the duality rotation is a local transformation in terms of the gauge fields $A^{a}$.

\footnotetext{
${ }^{3}$ Using a "formal" path integral quantization ("formal" in the sense that the possible UV divergences due to the nonGaussian character of the integral were not considered) it has been proved in 13] that the partition function of the PST and the Maxwell theories are equal. The absence of anomalies and non-trivial counterterms has been shown recently in 14. On this basis, it seems that the PST model can be trusted also at the quantum level.
} 


\section{M-theory viewpoint}

New insights on EM-duality symmetry have been provided by M-theory. Type IIB string theory reduced on a circle is known to be T-dual to M-theory on a torus. Accordingly, the S-duality symmetry of the IIB string theory is a consequence of the invariance of the torus under large diffeomorphisms, the $S L(2, \mathbb{Z})$ symmetry of the IIB theory being associated with the modular group of the torus. Thus, in the M-theory context the S-duality of IIB string theory arises elegantly from simple geometric arguments. Likewise, the system of a single M5-brane system provides an appealing geometric understanding of EM-duality symmetry.

The worldvolume of the M5-brane supports a self-interacting chiral two-form potential which couples minimally to dyonic strings located at the intersection of the M5-brane and some M2-branes ending on it. If the M5-brane is wrapped around the torus, the T-dual picture in IIB theory is a D3-brane with fundamental strings ending on it. As a consequence the D3-brane itself is inert under the modular group $S L(2, \mathbb{Z})$. In terms of the D3-brane worldvolume theory, this symmetry translates into the EM-duality symmetry of Abelian Born-Infeld theory [15]. This shed some light on the link between dualitysymmetric theories and chiral forms. Indeed, the e.o.m. (5) in four dimensions finds its origin in the self-duality equation of the three-form field strength living on the wrapped M5-brane. A $\mathbb{Z}_{2}$-duality transformation then corresponds to the exchange of the two circles in the compactification from $\mathrm{M}$ to IIB theory.

The next step of interest is to consider a system where several, say n, wrapped M5branes coincide. Unfortunately little is known about this interacting $(2,0)$ superconformal theory円. In the T-dual picture, the M5-branes appear as a set of coinciding D3-branes. Their dynamics is governed by a four-dimensional $U(n)$ supersymmetric Dirac-Born-Infeld theory which, in the weak field limit, is an ordinary $U(n)$ non-Abelian gauge theory with $\mathcal{N}=4$ supersymmetry. The determination of all higher order terms in $\alpha^{\prime}$ is still an open question but some progress have been made recently in this direction (see the talks of M. de Roo, A. Santambrogio and P. Koerber at this meeting). In any case, from the same arguments as before, the non-Abelian gauge theory on the coinciding D3-brane worldsheets should possess an $S L(2, \mathbb{Z})$ symmetry?.

Turning back to the eleven dimensional picture, the coinciding D3-branes dynamics suggests that a non-Abelian extension of the chiral two-form should exist. But the analysis of [17] shows that the standard Noether procedure will not provide such a theory as a local deformation of the free one. The interacting theory could fall outside the scope of perturbative covariant local field theory [16]. The same could be expected to occur for $S L(2, \mathbb{Z})$ duality symmetry of four dimensional non-Abelian vector theory, as we will explain in the following section.

\section{No-go theorems}

To conclude, let us consider the sourceless Yang-Mills equations and look after any duality symmetry property. They read

$$
D_{A}\left(\begin{array}{c}
F \\
* F
\end{array}\right)=0
$$

\footnotetext{
${ }^{4}$ Deep analogies are conjectured to occur for the $(4,0)$ superconformal theory 16 .

${ }^{5}$ This conjectured duality symmetry could be a new constraint to be imposed in order to derive the full non-Abelian Born-Infeld action.
} 
where $A$ is Lie-agebra valued one-form, its curvature is $F=D_{A} A=d A+A^{2}$ and the covariant derivative acts as $D_{A}=d+[A$,$] . Naively, one could think that the Yang-Mills$ equations (7) are left invariant by the duality rotation (3). However, that is not the case since the covariant derivative $D_{A}$ depends on $A$, which is not inert under the duality rotation.

In the seventies, two no-go theorems [9, 18] were found to prevent such a trivial generalization of EM-duality for Abelian gauge fields

Theorem 1 Generically, there is no infinitesimal transformation $\delta A$ which is able to implement the infinitesimal duality rotation

$$
\delta F=* F \delta \alpha, \quad \delta * F=-F \delta \alpha .
$$

Theorem 2 There exists one gauge field $A$ solution of $D_{A} * F=0$ such that there is no gauge field $\tilde{A}$ which is the "dual" of $A$, in the sense that $* F=D_{\tilde{A}} \tilde{A}$.

The second no-go theorem teaches us that the Poincaré lemma does not generalize straightforwardly to covariant derivative $D_{A}$ (Anyway, $D_{A}$ is not nilpotent since $D_{A}^{2}=[F$, ]). This prevents a direct application of the scheme of the section 3 to obtain a duality-symmetric formulation of Yang-Mills theory.

Of course, no-go theorems have the weakness of their hypotheses. In consequence, a priori nothing prevents less trivial generalizations of EM-duality symmetry for nonAbelian gauge theories. The following no-go theorem [0] further restricts the generalization possibilities 20]

Theorem 3 No consistent, local interactions of a set of free Abelian vector fields can deform the Abelian gauge transformations if the local deformed action (free action + interaction terms) continuously reduces to a sum of free, duality-symmetric, non-covariant actions in the zero limit for the coupling constant.

Since the two main assumptions of the theorem are locality and continuity of the deformations, in order to escape its conclusion and to correctly describe non-Abelian gauge field duality, one should perhaps leave the standard formalism of perturbative local field theory"

\section{Acknowledgments}

X.B. is grateful to the organizers for this very enjoyable meeting and he thanks N. Boulanger and G. Barnich for their remarks concerning the presentation and content of the talk. X.B. wish also to thank R. Argurio and G. Bonelli for discussions. This work was supported in part by the "Actions de Recherche Concertées" of the "Direction de la Recherche Scientifique - Communauté Française de Belgique", by IISN - Belgium (convention 4.4505.86) and by the European Commission TMR programme HPRN-CT2000-00131, in which X.B. is associated to Leuven.

\footnotetext{
${ }^{6}$ The proof of the third theorem is essentially algebraic and is based on a cohomological reformulation of the Noether procedure for constructing consistent deformations 19 .

${ }^{7}$ We stress that analogous theorems hold for any duality-symmetric theories in twice even dimensions.

${ }^{8} \mathrm{~A}$ definition of non-Abelian duality has been proposed in terms of loop space variables, which are intrinsically non-local. For recent reviews on this proposal, see for instance [21].
} 


\section{References}

[1] R. Penrose, Bull. Inst. Math. Appl. 10 (1974) 266.

[2] E. Schrödinger, Proc. Roy. Soc. (Lond) A150 (1935) 465;

G. W. Gibbons and D. A. Rasheed, Nucl. Phys. B454 (1995) 185 hep-th/9506035;

Phys. Lett. B 365 (1996) 46 [hep-th/9509141].

[3] M. K. Gaillard and B. Zumino, Nucl. Phys. B193 (1981) 221.

[4] P. A. Dirac, Proc. Roy. Soc. Lond. A 133 (1931) 60; Phys. Rev. 74 (1948) 817;

J. S. Schwinger, Phys. Rev. 173 (1968) 1536;

D. Zwanziger, Phys. Rev. 176 (1968) 1489.

[5] S. Deser, A. Gomberoff, M. Henneaux and C. Teitelboim, Phys. Lett. B 400 (1997) 80 [hep-th/9702184; Nucl. Phys. B 520 (1998) 179 [hep-th/9712189].

[6] C. M. Hull, JHEP 0109 (2001) 027 hep-th/0107149.

[7] J.H. Schwarz and P.C. West, Phys. Lett. B 126 (1983) 301;

P.S. Howe and P.C. West, Nucl. Phys. B 238 (1984) 181;

J. H. Schwarz, Phys. Lett. B 360 (1995) 13 [Erratum-ibid. B 364 (1995) 252] [hepth/9508143].

[8] D. Zwanziger, Phys. Rev. D3 (1971) 880.

[9] S. Deser and C. Teitelboim, Phys. Rev. D13 (1976) 1592.

[10] J.H. Schwarz and A. Sen, Nucl. Phys. B 411 (1994) 35.

[11] B. McClain, F. Yu and Y. S. Wu, Nucl. Phys. B 343 (1990) 689;

N. Berkovits, Phys. Lett. B 395 (1997) 28 [hep-th/9610134.

[12] P. Pasti, D. Sorokin and M. Tonin, Phys. Rev. D52 (1995) 4277 hep-th/9506109;;

Phys. Lett. B352 (1995) 59 hep-th/9503182.

[13] X. Bekaert and S. Cucu, JHEP0101 (2001) 015 [hep-th/0010266].

[14] O. M. Del Cima, O. Piguet and M. S. Sarandy, Nucl. Phys. B 600 (2001) 387 hepth/0012067.

[15] D. Berman, Phys. Lett. B409 (1997) 153 [hep-th/9706208];

A. Nurmagambetov, Phys. Lett. B436 (1998) 289 [hep-th/9804157.

[16] C. M. Hull, Nucl. Phys. B 583 (2000) 237 hep-th/0004195]; Class. Quant. Grav. 18 (2001) 3233 [hep-th/0011171]; JHEP 0012 (2000) 007 [hep-th/0011215.

[17] X. Bekaert, M. Henneaux and A. Sevrin, Phys. Lett. B 468 (1999) 228 hepth/9909094]; Nucl. Phys. Proc. Suppl. 88 (2000) 27 [hep-th/9912077].

[18] C. Gu and C. Yang, Sci. Sin. 18 (1975) 483.

[19] G. Barnich and M. Henneaux, Phys. Lett. B 311 (1993) 123 [hep-th/9304057.

[20] X. Bekaert and S. Cucu, Nucl. Phys. B 610 (2001) 433 hep-th/0104048. 
[21] H. M. Chan and S. T. Tsou, Int. J. Mod. Phys. A14 (1999) 2139, hep-th/9904102;

H. M. Chan, J. Bordes and S. T. Tsou, Int. J. Mod. Phys. A 14 (1999) 2173 [hep$\mathrm{ph} / 9809272$

H. M. Chan, Int. J. Mod. Phys. A 16 (2001) 163 [hep-th/0007016]. 